\title{
Patent Ductus Arteriosus Device or Surgical Closure: the Role of Charity Missions Where the Resources Are Limited
}

\author{
Abdallah Elamin Elsheikh \\ Medani Heart Centre \\ Salah Eldin M.E.HASSAN \\ Medani Heart Centre \\ Abdal Mahmoud El Siddig \\ Medani Heart Centre \\ Mohamed Elamin Ahmed \\ Medani Heart Centre \\ Sabir Taha Hussein \\ Medani Heart Centre \\ Mohammed Mahmmoud Fadel Allah ELjack ( m.mahmmoud96@gmail.com ) \\ Medani Heart Centre https://orcid.org/0000-0002-2370-9368
}

Research article

Keywords: patent ductus arteriosus (PDA), charity missions, expensive devices

Posted Date: November 5th, 2021

DOI: https://doi.org/10.21203/rs.3.rs-959615/v2

License: () (1) This work is licensed under a Creative Commons Attribution 4.0 International License.

Read Full License 


\section{Abstract}

Objectives: To compare the different methods of treatment of patent ductus arteriosus (PDA), and evaluate the role of charity missions in availing the expensive devices for needy patients where the resources are limited.

Background: The role of surgery is gradually being taken over by catheter intervention, however the cost in not affordable to most of our patients. Charity organizations are helping limited resource countries by availing the devices.

Methods. All paediatrics patent ductus arteriosus (PDA) patients presenting to Medani Heart Centre $(\mathrm{MHC})$ in a 6-year period were included in this study. The study included 337 patients. 283 were treated by catheter intervention group (A) and 54 were treated surgically group (B), 22 patients were referred for surgery from the catheterization laboratory, and 32 were treated when there was no visiting teams.

Results: A total of 337 patients were included in this study, 32 were treated surgically by the local surgical teams. Out of remaining 305 patients 283 were found suitable for catheterization intervention, and were treated successfully, 22(\%7) were having other congenital anomalies or PDA not suitable for intervention and were referred to surgery. 54 patients were treated by surgical ligation, 22 were referred from the intervention group and 32 were treated surgically when there were no visiting delegates and patients' condition dictate urgent intervention, with good results. Devices were provided by charity organizations and delivered by the visiting teams, the choice of device depends on the availability.

Conclusions: Surgery remains a good modality of treatment were the resources are scares, however a number of charity organizations are offering these expensive devices for countries of low and middle income making it possible for the poor patients to have such treatment. The results of both treatments are excellent

\section{Introduction}

The ductus arteriosus is responsible for carrying $55-60 \%$ of total fetal output during intrauterine life and is usually closed within 2 to 3 weeks of age after birth. The persistence of blood flow through this channel after this age is a defect, which is responsible for $7-11 \%$ of all congenital cardiac defects (1). Once significant patent ductus arteriosus (PDA) is present, closure is always indicated to prevent associated complication such as pulmonary hypertension, arrhythmias, infective endocarditis, aneurysm formation and heart failure (2). Surgical ligation of PDA was 1st described by E. Gross in 1930s through lateral thoracotomy with good results and the later transcatheter PDA closure was introduced by Portsmann et al. in 1967, there have been many significant developments in the devices used to close a PDA. In the past 20 years, transcatheter closure has become the leading approach to closure of most PDAs (3).

\section{Material And Methods}


All patents admitted to Medani Heart Center (MHC) in Wadmedni_Gezira state_Sudan in the period of study were included in this retrospective hospital based study. All patients were diagnosed by Echocardiography Vivid-7 system (GE Medical System, Milwaukee, WI, USA). by a single operator ${ }^{2}$ the diagnosis is confirmed by the cardiologists in the visiting delegates in the day of the intervention. All patients were planned for catheter intervention which is done by different charity missions visiting the center during the study period. Those patients with large PDAs or when the size of the device selected is not available, were referred to surgery which is done by the local surgical staff or the guest surgeons. During the period between February 2011 and December 2016,339 pediatric PDA patients age 70 days to 17 years (table 1) were treated, (in2 patients the PDA was found too tiny to deserve closure) mean age (3.8years) mean weight $(11.9 \mathrm{Kg}) .129$ were males, 210 were females, M: $\mathrm{F}$ ratio 1:1.6. 285 patients were treated by catheter (group A) mean age 3.9 and 54 were treated by surgical ligation (group B) mean age (3.2), this group was composed from 32 patients treated when there were no visiting teams, and 22 patients were referred to surgery after a failed attempt to close in the catheterization laboratory; For unfavorable anatomy $(n=2)$ unavailability of suitable device size $(n=3)$ difficult access $(n-1)$ concomitant congenital anomaly (sub aortic membrane $(n=2)$ surgically resected, secondum atrial septal defect (ASD), $(n=2)$,closed surgically, severe pulmonary stenosis(PS), $(n=2))$ and huge PDA in the others.

3 patients were down syndrome, 1 Marfan syndrome, one congenitally deaf, and one patient with an imperforate anus having a colostomy.

In group (A) out of 285 patients 283 patients were treated by catheter intervention in the catheterization laboratory, (cath.lab.) in 2 patients the PDA was found too tiny to deserve closure. Catheterization Closure was done using device in 249, Amplatzer Duct Occluder (ADO, AGA Medical Corporation, Golden Valley, Minnesota) $n=169)$, Lifetech ( $n=45)$, and occlutech (La Cours Gata Sweden) ( $n=37)$, and Flipper coils (Cook Medical) ( $\mathrm{n}=34)$. and a 54 patients were treated surgically (group B). All patients were checked for residual leaks in the cath. lab. And in first post-operative day by echo. Those treated by catheter intervention were discharged the next morning after the echo check, (mean hospital stay 10 hours). For group (B) surgery was performed in the operation theatre (OR) by the local surgical staff under general anesthesia through a left lateral thoracotomy through $3^{\text {rd }}$ intercostal space and the PDA double or triple ligated with heavy silk suture, a chest drain was routinely left in the left plural space, patients were then transferred to the surgical ICU and mechanically ventilated for 2 to 3 hours and transferred to the surgical ward in the first post-operative day and discharged home on the third or fourth post-operative day (mean 3.2 days) after checking for residual leak by echo.

\section{Results}

All patients in group (A) were taken to cath.Lab. with the intension to treat. Out of 307 , in two patients the PDA was found too tiny to deserve intervention. The procedure was successful in 283 out of $305(93 \%)$ while in $22(7 \%)$ the procedure was abandoned and patients referred to surgery. 32 patients were treated surgically when there were no visiting teams, in addition to those referred from cath.lab. There were no deaths or major complications in both groups. The coil migrated into the left pulmonary artery in one 
patient and was removed in the operation theatre and the PDA was ligated. In two patients the device migrated into the descending aorta and removed from the femoral artery with catheter without the need to interfere surgically. One patient in group A (aged 2years) had a cardiac arrest intubated and was admitted to the ICU ventilated for few hours recovered.3(1\%) patients in group (A) and 13(24\%) patients in group (B) received blood transfusion. ICU stay and hospital stay were longer in surgical group. $(p=-)$. Blood

All patients in group(A) were checked in the cath. Lab. And on the first postoperative day by echocardiography for leaks and if there were no residual PDAs patients were discharged. In group (B) the ductus was checked digitally in the (OR) for residual thrill and in the first post-operative day by echo.

In group A there was minimal leak $(n=10)(3.5 \%)$ detected in the cath. Lab. That completely disappeared in the postoperative echo. There was no significant leak in all surgically treated patients.

\section{Discussion}

PDA closure leads to normal life expectancy of patient equal to normal population (1), and since the first successful surgical closure by Gross in 1930 surgery remained the treatment of choice, till Portsmann introduced the trans-catheter closure of the PDA in 1967, and since then a variety of devices and Occluders were tried with improving results over time. In this era of trans-catheter intervention for PDA treatment, surgery is reserved for the premature and low body weight patients and for complication of trans-catheter intervention or when the PDA is too big so that the device may protrude into descending aorta, however in countries with limited resources, surgery is a good choice as the new devices are very expensive

In our study all patients were scheduled for catheter intervention and remain in waiting lists for visiting charity mission from Saudi Arabia and other gulf countries. Symptomatic patients who cannot wait are treated surgically by local staff. During charity missions all are taken to catheterization laboratory. with intension to treat. PDA associated with other congenital heart anomalies that deserve treatment were treated surgically. Providing devices for closure of PDA by charity organizations made it possible in our situation of limited resources to offer the service for the needy. Catheterization therapy in pediatric PDA patients did not show a significant advantage in primary success rate, total complications, or blood transfusion (5) but it was associated with reduction in length of hospital stay $(4,5,8,11.12)$ it is effective and less invasive method as compared to the surgical ligation, but the cost is much higher as compared to surgical ligation $(1,5)$. However Zulqarnain et al $(1)$ reported low cost of trans-catheter closure using The SHSMA occluder (Shanghai Shape Memory Alloy Company). Residual leak in early reports of catheter intervention was high. Hawkins et al (6) reported a rate as high as $25-35 \%$ that reduced to about $4 \%$ on follow up, the results continued to improve over time so that recent studies report residual shunts between $0-2 \%(1,8,9)$. Pass et al $(10)$ reported a success rate of $99 \%$ in335 out of 339 patients. Darryal T.et al stated that surgical ligation of PDA is more effective and less expensive and superior to placing an Occluder (6). In our study there was minimal leak in 10 patients of group (A) (3.5\%) detected in the cath. 
Lab. That disappeared completely on the echo check before discharge, there was no leak in group (B). In contemporary practice, percutaneous device closure is an effective and safe treatment option for children, adolescent and adult PDA patients $(3,12)$. The incidence of residual shunting in surgical group was $0 \%$ in this study. Many other studies have reported that the incidence of residual shunting in surgical closure of PDA varies from 1.5 to 23 (6) specially when a single tie was used, in our study we used double ligation or triple ligation which may explain our results.

\section{Conclusions}

Closure of PDA using devices and coils or by surgical ligation results in good outcome. Results of device closure has improved over the years resulting in excellent outcomes, but the high cost remains a problem in places with limited resources. Charity missions play a crucial role in providing this service with comparable results with the international literature. Surgical ligation remains a good alternative when there are no visiting teams, with good results and affordable cost.

\section{Declarations}

\section{Ethical Approval:}

Ethical Approval obtained from Research and Ethics committee,Medani Heart Centre.

\section{Consent to participate:}

Verbal and written consents were obtained from patients before writing the case or using informations.

\section{Consent For Publication:}

Written consent to publish this information was obtained from the patient.

\section{Competing of interests:}

The authors have no conflict of interst to declare

\section{Funding:}

The Study was funded by the authors themselves.

\section{References}

1. Zulqarnain A, Younas M, Waqar T, Beg A, Asma T, Baig MAR. Comparison of effectiveness and cost of patent ductus arteriosus device occlusion versus surgical ligation of patent ductus arteriosus. Pak J Med Sci. 2016;32(4):974-977. doi: http://dx.doi.org/10.12669/pjms.324.10048

2. Nady ME, Amrousy DE, Salah N and Zoair A. Transcatheter Versus Surgical Closure of Patent Ductus Arteriosus in Pediatric Patients: A Systematic Review With Meta-Analysis. SM J Pediatr Surg. 2017; 
3(4): 1054

3. Alban-Elouen Baruteau, Sébastien Hascoët, Julien Baruteau , Younes Boudjemline ,Virginie Lambert, Claude-Yves Angel, Emre Belli ,Jérôme Petit, Robert Pass, Transcatheter closure of patent ductus arteriosus: Past, present and future. Archives of Cardiovascular Disease (2014) 107, 122-132

4. Kai Wang, M D; Xuanren Pan, M D; Qiaoyun Tang, MD; Yusheng Pang, M D, PhD. Catheterization Therapy vs Surgical Closure in Pediatric Patients With Patent Ductus Arteriosus: A MetaAnalysis. Clin. Cardiol.37,3,188-194(2014)

5. Costa et al. Cost-Effectiveness of Surgical vs. Percutaneous Treatment of PDA Rev Bras Cardiol Invasiva.2014;22(2):168-79

6. Hawkins et al Cost and efficacy of surgical ligation versus transcatheter coil occlusion of patent ductus arteriosus. J.thorac. cardio. Surg. 112,(6) 1634-1640.

7. Darryl T.etal Clinical outcomes and cost of transcatheter as compared with surgical closure of patent ductus arteriosus, n.eng.j.med.Vol 329,no21,(1993)

8. Thanopoulos et al PDA Closure with the Amplatzer Duct Occluder JACC Vol. 35, No. 4, 2000 March $15,2000: 1016-21$

9. El-Said et al Adverse Events Associated With PDA Closure J Am Heart Ass oc.2013;2 :e000424 doi:10. 1161/JAHA.113.000424)

10. Pass et al USA Amplatzer PDA Occlusion Device Trial JACC Vol. 44, No. 3, 2004 August 4, 2004:5139

11. Sudhakar P, John Jose, Oommen K. George. Contemporary outcomes of percutaneous closure of patent ductus arteriosus in adolescents and adults Indian Heart Journal 70 (2018) 308-315

12. KHAN ET AL EMBOLIZED AMPLATZER DUCT OCCLUDER TO AORTA: RETRIEVA

13. LTECHNIQUE J Saudi Heart Assoc. 2016;28:116-118

\section{Tables}

\section{Table (1)}

\begin{tabular}{|lllll|}
\hline Patients and procedure & Total $(\mathrm{n}=339)$ & Device(249) & Coil(34) & Surgery(54) \\
\hline Age & Total(F) & Total(F) & Total(F) & Total(F) \\
\hline$\square 6$ mon. & $50(34)$ & $26(12)$ & $6(2)$ & $9(7)$ \\
\hline 6 mon to 2y & $143(85)$ & $103(67)$ & $10(5)$ & $21(12)$ \\
\hline $2 y$ to 6 y & $82(47)$ & $68(31)$ & $9(7)$ & $18(10)$ \\
\hline$\square 6 y$ & $62(44)$ & $52(32)$ & $9(7)$ & $6(3)$ \\
\hline
\end{tabular}

Patients Demographic Data and Procedures ( 2 patients were not treated because of tiny PDA) 
Table (2)

\begin{tabular}{|lllll|}
\hline No & Diagnosis & Mode of treatment & outcome & Remarks \\
\hline 7 & VSD & 5 Device closure & No leak & 2 VSD Surgical \\
\hline 2 & ASD & Device closure & No leak & ASD Device Cl. \\
\hline 2 & SAM & 1 device 1SL & No leak & SAM resection \\
\hline 3 & PS & 1 device 1 coil & No leak & 1 PAP \\
\hline 2 & CoArct & Device & No leak & Mild coarct. \\
4 & Pul. HTN & device & No leak & Pul P. droped \\
\hline
\end{tabular}

Associated congenital anomalies. VSD (Ventricular Septal Defect), ASD (Atrial Septal Defect), SAM (Sub Aortic Membrane), PS (Pulmonary Stenosis), CoArct (Coarctation of the aorta), Pul HTN (Pulmonary Hypertension) 